\title{
CXCL-8 Regulates Head and Neck Carcinoma Progression through NOD Signalling Pathway
}

\author{
Leong-Perng Chan ${ }^{1,2}$, Cheng Liu ${ }^{3,4}$, Ya-Ping Tseng ${ }^{5}$, Pin-Ju Chen ${ }^{6}$, Da-Long Cheng ${ }^{7}$, Wan-Ting \\ Chen ${ }^{6}$ and Chia-Hua Liang ${ }^{6, a}$ \\ ${ }^{1}$ Institute of Clinical Medicine, Kaohsiung Medical University, Kaohsiung, Taiwan \\ ${ }^{2}$ Department of Otolaryngology-Head and Neck Surgery, Kaohsiung Medical University Hospital, Kaohsiung Medical \\ University, Kaohsiung, Taiwan \\ ${ }^{3}$ Division of Plastic Surgery \& HBOT Center, Chi Mei Medical Center, Tainan, Taiwan \\ ${ }^{4}$ Department of Electrical Engineering, Southern Taiwan University of Science \& Technology \\ ${ }^{5}$ Institute of Basic Medical Sciences, National Cheng Kung University, Tainan, Taiwan \\ ${ }^{6}$ Department of Cosmetic Science and Institute of Cosmetic Science, Chia Nan University of Pharmacy and Science, \\ Tainan, Taiwan \\ ${ }^{7}$ Department of Computer and Communication, Shu-Te University, Kaohsiung, Taiwan
}

\begin{abstract}
Head and neck squamous cell carcinoma (HNSCC) ranks sixth among the most common cancers in the world. Interlukin-8 (CXCL-8), a major role in inflammatory response and tumor microenvironment, correlates with tumor progression, metastasis and invasion. We explored CXCL-8 promotes tumor progression in different differentiation HNSCC cells. This project would apply to development on biomarker and target in HNSCC as well as provide a basis of early diagnosis and treatment for clinical. CXCL-8, NOD1 (nucleotide-binding oligomerization domain-containing protein 1) and receptor-interacting protein kinase (RIPK2) levels were detected statistically higher in patient tissue with HNSCC than in non-cancerous matched tissue (NCMT) in the microarray and qRT-PCR study, whereas NOD2 was weakly expressed. Similar results were obtained for CXCL-8, NOD1, NOD2 and RIP2 from RT-PCR and western blotting. High CXCL-8, NOD1 and RIP2 expressions were found on HNSCC patient tissue than that of NCMT, whereas NOD2 was weakly expressed. The analytical results indicate that CXCL-8 is required in NOD 1-mediated signalling pathways in HNSCC.
\end{abstract}

\section{Introduction}

Head and neck squamous cell carcinoma (HNSCC) is the sixth most common cancer in the world. Oral cancer, predominantly oral SCC (OSCC), is a high-impact disease of the oral cavity. OSCC is generally detected in the late stages when the cancer has advanced, and therefore has a poor prognosis and survival rate [1]. Surgery and radiotherapy are currently the primary treatments, but they typically cause postoperative defects and functional impairments in patients owing to the location of OSCC in the head and neck [2]. Since morbidity and mortality rates associated with HNSCC have improved very little over the past 30 years, early detection or prevention of the disease is likely to be most effective. Therefore, basic research into HNSCC is increasing, focusing on the identification of specific biomarkers for the diagnosis of its nature and aggressiveness [3]. Cytokines are regulators of

\footnotetext{
${ }^{\mathrm{a}}$ Corresponding author: tinna_ling@mail.cnu.edu.tw
} 
host responses to infection, immune responses, inflammation, and trauma. Some cytokines make disease worse (pro-inflammatory), whereas others reduce inflammation and promote healing (antiinflammatory) [4]. HNSCC is known to develop many molecular strategies to escape from efficient anti-tumor immune responses. Researchers do not yet well understand the origin of the signals and mechanisms underlying these responses. However, based on the local and systemic nature of these responses, researchers hypothesize that SCC produces cytokines with pro-inflammatory, proangiogenic and immune-regulatory activities possibly contributing to the pathogenesis of HNSCC [5]. Interleukin-8 (CXCL-8) plays a significant role in inflammatory response and tumor microenvironment; it correlates with tumor progression, metastasis and invasion, and could thus contribute to the pathogenesis of the disease [1]. Blocking IL-1, CXCL-8 or TNF has been highly successful in patients with rheumatoid arthritis, inflammatory bowel disease or graft-vs-host disease [6]. Previous research has indicated that CXCL-8 is a potential biomarker for HNSCC in bodily fluids such as blood and saliva [7]. However, different studies have found wide variations the CXCL-8 levels, and have not measured all tissue CXCL-8 levels. The NOD pathways directly regulate cytokine release, particularly of pro-inflammatory cytokines, such as IL- $1 \beta$, IL-6, CXCL-8 and other immune response regulating cytokines such as IL-10 or TNF- $\alpha$ [8]. NLRs are expressed in various cell types, including macrophages, neutrophils, epithelial and endothelial cells, dendritic cells, and in malignant tumors, including melanoma and HNSCC [9]. CXCL-8 has a significant role in inflammatory responses in a tumor microenvironment; it affects tumor progression, metastasis and invasion, and so contributes to the pathogenesis of the disease [1]. CXCL-8 is responsible for most of the angiogenic activity that is induced by human OSCC [10]. Recent studies have suggested that nucleotide binding oligomerization domain (NOD)-like receptors (NLRs) are key modulators in inflammatory diseases [11-12]. A previous study revealed that the expression profile of NLRs in HNSCC cells differed from that in healthy epithelial cells. Stimulation by NOD1 induced an immunological response in tumor cells that differed from that in normal epithelial cells [13]. The mechanism of the CXCL-8-induced NOD pathway from HNSCC and its regulation are poorly understood. Therefore, identifying its role in tumorigenesis can lead to novel treatment modalities for HNSCC.

\section{Methods and materials}

\subsection{Tissue samples}

All tissue samples were fresh frozen in liquid nitrogen until RNA and protein purification and microarray experiments and collected with patient consent under approval of the institutional review boards from all participating institutions. For Immunohistochemistry, HNSCC and non-cancerous matched tissue (NCMT) tissue were fixed in buffered formalin and embedded in paraffin, sliced into 3 $\mu \mathrm{m}$ sections, and mounted on glass slides. For RNA and protein isolation, HNSCC and NCMT tissue were placed into liquid nitrogen for further biochemical analyses. Flash-frozen tissue was removed from the freezer and placed into the mortar. Tissue was ground to a fine powder and scraped into a liquid nitrogen-cooled microcentrifugetube.

\subsection{Microarray}

For the expression profiling of HNSCC and NSCT, total tissue RNA was extracted using Trizol reagent (Invitrogen, Carlsbad, CA, USA). An RNeasy Mini Kit (Qiagen, Hilden, Germany) was used to quantify the isolated. RNA isolated at OD $260 \mathrm{~nm}$ and a bioanalyzer (Agilent Technology, USA) was used to conduct a qualitative. A $0.2 \mathrm{~g}$ mass of total RNA was amplified using a Low-Input QuickAmp Labeling Kit (Agilent Technologies, USA) and labeled with Cy3 (CyDye, Agilent Technologies, USA) during the in vitro transcription process. A $0.6 \mathrm{~g}$ mass of Cy3-labled cRNA was fragmented to a men size of approximately 50-100 nucleotides by incubation with fragmentation buffer at $60^{\circ} \mathrm{C}$ for 30 minutes. Correspondingly fragmented labeled cRNA was then pooled and hybridized using an Agilent 
Sure Print G3 Human V2 GE $8 \times 60 \mathrm{~K}$ Microarray (Agilent Technologies, USA) at $65^{\circ} \mathrm{C}$ for $17 \mathrm{~h}$. After they were washed and dried by blowing with a nitrogen gun, the microarrays were scanned using an Agilent microarray scanner (Agilent Technologies, USA) at $535 \mathrm{~nm}$ for Cy3. Scanned images were analyzed using Feature Extraction 10.5.1.1 software (Agilent Technologies, USA), which is image analysis and normalization software. The signal and background intensity were quantified for each feature.

\subsection{Quantitative real-time PCR (QRT-PCR) of CXCL-8 mRNA}

QRT-PCR analysis was evaluating the mRNA expression of CXCL-8 and NOD1 in HNSCC and NCMT in human tissue to verify the results for the microarray (Welgene Biotech. Co., Taipei, Taiwan).Total RNA was extracted using a Total RNA Miniprep Purification Kit (GeneMark). qRTPCR was conducted using specific primers. The amplification reactions were carried out in a $20 \mu 1$ mixture, using SYBR Green Supermix (Bio-Rad Laboratories). All PCRs were normalized to internal control $\beta$-actin mRNA.

\subsection{RT-PCR analysis}

For tissue RNA isolation, the RNA was extracted as Total RNA Miniprep Purification Kit (GeneMark). For cellular RNA extraction, RNAs were isolated from vehicle control or 10 and 100 $\mathrm{ng} / \mathrm{ml}$ concentrations of CXCL-8-treated cells for $72 \mathrm{~h}$ using the Trizol reagent (Invitrogen, Carlsbad, CA, USA). Single stranded cDNA was transcribed by priming with oligo-dT with SAMscript reverse transcriptase (GeneMark, Taipei, Taiwan). PCR amplification of the cDNA was done in a reaction mixture containing Taq polymerase (GeneMark, Taipei, Taiwan). The amplified RT-PCR products were electorphoresed on a $\%$ agarose gel, visualized by ethidium bromide staining and photographed under ultraviolet light. All results were normalized to the house-keeping $\beta$-actin gene. The obtained bands were visualized by image $\mathrm{J}$ software.

\subsection{Western blot analysis}

For tissue protein extraction, the frozen tissue were homogenized in lyses buffer $(50 \mathrm{mM}$ HEPES, $\mathrm{pH}$ 7.5, $150 \mathrm{mM} \mathrm{NaCl}, 10 \%$ glycerol, 1\% Triton X-100, 1mM EDTA, $1 \mathrm{mM}$ EGTA, $50 \mathrm{mM} \mathrm{NaF}, 1 \mathrm{mM}$ sodium orthovanadate, $30 \mathrm{mM}$-nitrophenyl phosphate, $10 \mathrm{mM}$ sodium pyrophosphate, $1 \mathrm{mM}$ phenylmethylsulfonyl fluoride, $10 \mu \mathrm{g} / \mathrm{ml}$ aprotinin, and $10 \mu \mathrm{g} / \mathrm{ml}$ leupeptin). Supernatants were collected and analyzed. Tissue extracts and cell lysates were prepared and subjected to western blotting with antibodies against CXCL-8, NOD1, NOD2, RIP2, CXCR1 and CXCR2, respectively, as described. Proteins were visualized with ECL reagent and their relative expression was determined by densitometry using ImageJ software program and normalized relative to $\beta$-actin.

\subsection{Statistical analysis}

The results are expressed as mean \pm standard deviation (SD). Statistical differences were estimated by one-way analysis of variance (ANOVA) followed by Dunnett's test or the Tukey-Kramer test. A p value of 0.05 was regarded as significant. The data were analyzed and the figures potted using software (SigmaPlot Version 8.0 and SigmaStat Version 2.03, Chicago, IL).

\section{Results and Discussion}

\subsection{Microarray, CXCL-8 and NOD signalling pathway}


This investigation assessed the effect of CXCL-8 on NOD signalling. A microarray analysis was first performed to consider the expression of innate immunity genes in HNSCC and NCMT of human tissue. Specifically, a significantly increased expression ( $>2$-fold) was observed in several genes in the inflammasome signalling pathway in HNSCC, including CXCL-8, NOD1, NOD2 and RIP2 as compared with NCMT. The factors of increase were 9.93 for CXCL-8, 3.14 for NOD1, 4.23 for NOD2 and 6.95 for RIP2 (Figure 1A). This relationship indicates that the upregulation of CXCL-8 correlates with the overexpression of NOD-mediated signalling in HNSCC. To examine the possible role of CXCL-8 and NOD as informative biomarkers of HNSCC, as indicated in the microarray study, the levels of CXCL-8, NOD1 and NOD2 in HNSCC and NCMT tissue $(n=6)$ were determined using qRT-PCR. As shown in Figure1B, the mean CXCL-8 and NOD1 level was detected statistically higher (3.70-foldand 2.12-fold, respectively) in patient tissue with HNSCC than in NCMT, whereas NOD2 was weakly expressed. These results confirmed the use of CXCL-8 and NOD1 as biomarkers for HNSCC detection with microarray analysis (Figure 1A). Thus, experimental data suggest that CXCL-8 and NOD1 had higher expression in HNSCC than in NCMT, whereas NOD2 was absent.

\begin{tabular}{cccc} 
A & & & \\
\hline Gene symbol & Gene name & Signal in NCMT & Signal in HNSCC \\
\hline CXCL-8 & interleukin 8 & 173.865 & 1726.490 \\
NOD1 & nucleotide-binding oligomerization domain containing 1 & 1189 & 3730 \\
NOD2 & nucleotide-binding oligomerization domain containing2 & 107.840 & 456.603 \\
RIPK2 & receptor-interacting serine-threonine kinase 2 & 1271.418 & 8839.061 \\
\hline
\end{tabular}

B

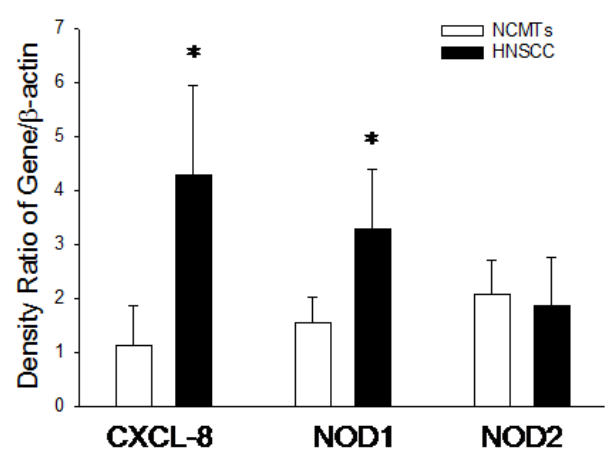

Figure 1. The expression of CXCL-8 and NOD signaling pathway in NCMT and HNSCC of human tissue. A microarray analysis to assess the expression (fold change threshold >2) of CXCL-8, NOD1, NOD2 and RIP2 in NCMT and HNSCC of human tissue. (B) The microarray data were substantially verified by qRT-PCR: CXCL-8, NOD1 and NOD2 were upregulated in HNSCC, as compared with NCMT tissue $(n=6) ;{ }^{*} p<0.05$ indicates a significant difference from NCMT.

\subsection{CXCL-8 and NOD1 activation in HNSCC}

NOD1 induces inflammation by stimulating cellular signalling pathways through the adaptor molecule RIP2. This process in turn induces NF- $\mathrm{B}$ as well as p38 and JUN amino-terminal kinases (JNK) MAP kinases. To confirm and complement the mRNA data, RT-PCR (Figure 2A) and western blotting (Figure 2B) also confirmed that the CXCL-8 and NOD pathway-related gene and protein expressions (NOD1 and RIP2) were higher in HNSCC tissue than in NCMT. Similar results were obtained for CXCL-8, NOD1, NOD2 and RIP2 from RT-PCR and western blotting. High CXCL-8, 
NOD1 and RIP2 expressions were found on HNSCC patient tissue than that of NCMTs, whereas NOD2 was weakly expressed. Additionally, immunohistochemical staining was performed to demonstrate the morphological localization of the CXCL-8, NOD1 and RIP2.

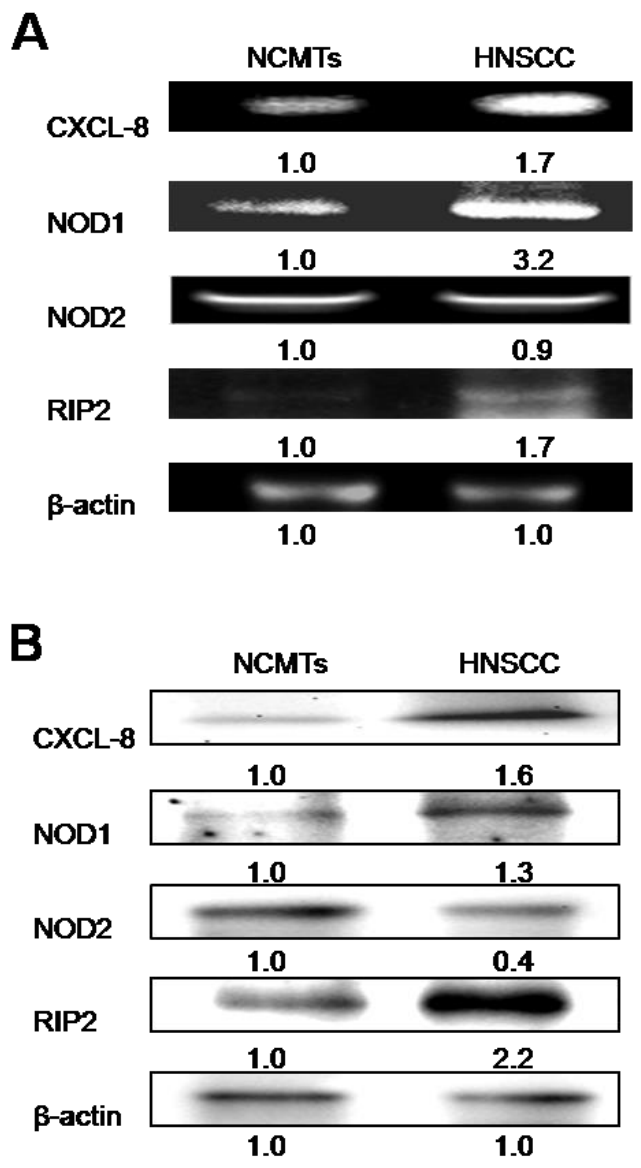

Figure 2. CXCL-8 and NOD signalling pathway in HNSCC. CXCL-8, NOD1, NOD2, RIP2 expressions in NCMT and HNSCC of human tissue were analyzed by RT-PCR (A) and western blotting (B). Quantities of $\beta$ actin were adopted as internal controls for sample loading.

\section{Conclusions}

Previously reports have demonstrated salivary IL-8 levels in oral lichen planus (OLP) patients are significantly decreased after dexamethasone treatment, which indicates that the levels of IL- 8 could be influenced by the degree of inflammation in OLP. The present study was conducted to test the hypothesis, in the presence of chronic inflammation, irritation and carcinogenic exposure of oropharyngeal mucosa, IL-8 will be elevated leading to further activation of NOD signalling pathway contributing to a local environment which may be associated with progression of these lesions to human HNSCC. Since IL-8 play and important role in pathogenesis of HNSCC, and have been linked with increased tumor growth and metastasis, and we found that NOD pathway-mediated protein was increased by IL-8 stimulation in HNSCC cells. Our results demonstrate that IL-8 not only enhances in HNSCC tissue, but also have a stimulatory effect on the activity of NOD pathway. Further validation studies may need to be performed using a larger sample cohort for a greater statistical comparison between HNSCC and healthy control groups, which should be included to validate firmly IL-8 and 
NOD1 as biomarkers for HNSCC. Therefore, if its role in tumorigenesis can be sufficiently assessed, it could open up new avenues to fine out novel treatment modalities for HNSCC.

\section{References}

1. M.A. John, Y. Li, X. Zhou, P. Denny, C.M. Ho, C. Montemagno, W. Shi, F. Qi, B. Wu, U. Sinha, R. Jordan, L. Wolinsky, N.H. Park, H. Liu, E. Abemayor, D.T. Wong. Arch Otolaryngol Head Neck Surg. 8 (2004)

2. P.J. Thomson, J. Wylie. Int J Oral Maxillofac Surg. 2 (2002)

3. M.E. Arellano-Garcia, S. Hu, J. Wang, B. Henson, H. Zhou, D. Chia, D.T. Wong. Oral Dis. 8 (2008)

4. B.B. Aggarwal, S. Shishodia , S.K. Sandur, M.K. Pandey, G. Sethi. Biochem Pharmacol. 11 (2006)

5. N.L. Rhodus, C.S. Miller, S. Myers, F. Ondrey. Cancer Detect Prev. 1 (2005)

6. S.E. Lee, J.M. Kim, S.K. Jeong, J.E. Jeon, H.J. Yoon, M.K. Jeong, S.H. Lee. Arch Dermatol Res. $10(2010)$

7. Y.S. Hwang, S.K. Lee, K.K. Park, W.Y. Chung. Oral Oncol. 1 (2012)

8. O.S. Shin, J.B. Harris. Korean J Hematol. 2 (2011)

9. F. Qiu, A. Maniar, M.Q. Diaz, A.I. Chapoval, A.E. Medvedev. Innate Immun. 4 (2011)

10. S.E. Lee, J.M. Kim, S.K. Jeong, J.E. Jeon, H.J. Yoon, M.K. Jeong, S.H. Lee. Arch Dermatol Res. $10(2010)$

11. S.R. Punyani, R.S. Sathawane. Clin Oral Investig. 2 (2013)

12. N.L. Rhodus, V. Ho, C.S. Miller, S. Myers, F. Ondrey. Cancer Detect Prev. 1 (2005)

13. Y.S. Cheng, L. Jordan, L.M. Gorugantula, E. Schneiderman, H.S. Chen, T. Rees. J Periodontol. 7 (2014) 